\begin{tabular}{|c|l|}
\hline Title & High-dimensional heteroclinic and homoclinic connections in odd point-vortex ring on a sphere \\
\hline Author(s) & Sakajo, Takashi \\
\hline Citation & $\begin{array}{l}\text { Nonlinearity, 19(1), 75-93 } \\
\text { https://doi.org/10.1088/0951-7715/19/1/005 }\end{array}$ \\
\hline Issue Date & 2006-01 \\
\hline Doc URL & http://hdl.handle.net/2115/5417 \\
\hline Rights & Copyright $\odot$ 2006 IOP Publishing Ltd. \\
\hline Type & article (author version) \\
\hline File Information & NL19-1.pdf \\
\hline
\end{tabular}

Instructions for use 


\title{
High-dimensional heteroclinic and homoclinic connections in odd point-vortex ring on a sphere
}

\author{
Takashi SAKAJO \\ Department of Mathematics, Hokkaido University, \\ Sapporo Hokkaido 060-8610, JAPAN. \\ Tel: +81-11-706-4660, Fax: +81-11-727-3705, \\ E-mail:sakajo@math.sci.hokudai.ac.jp.
}

July 3,2005

\begin{abstract}
We consider the motion of the $N$-vortex points that are equally spaced along a line of latitude on sphere with fixed pole vortices, called " $N$-ring". We are especially interested in the case when the number of the vortex points is odd. Since the eigenvalues that determine the stability of the odd $\mathrm{N}$ ring are double, each of the unstable and stable manifolds corresponding to them is two-dimensional. Hence, it is generally difficult to describe the global structure of the manifolds. In this article, based on the linear stability analysis, we propose a projection method to observe the structure of the iso-surface of the Hamiltonian, in which the orbit of the vortex points evolves. Applying the projection method to the motion of the 3-ring and 5-ring, we characterize the complex evolution of the unstable odd $N$-ring from the topological structure of the iso-surface of the Hamiltonian.
\end{abstract}

PACS: 47.32.Cc, 47.20.Ky, 05.45.-a

Keywords: Vortex points; Flow on a sphere; Heteroclinic manifold; Projection method

\section{Introduction}

In the mathematical study of fluid motions on Earth, we often assume that the incompressible and inviscid flow is confined to the surface of the sphere. Since the vorticity is conserved along the path of a fluid particle like two-dimensional flows, it is sufficient to consider the coherent local regions where the vorticity exists at the initial moment. The following element we need to introduce is the effect of rotation of the sphere. However, because of the Coriolis force due to the rotation, not the vorticity but the potential vorticity becomes the conserved quantity[1]. Accordingly, it is insufficient to consider only the coherent initial vortex structure, since the vorticity generates and disappears everywhere in the sphere during the evolution. Thus, in this article, instead of dealing with the Coriolis force directly, we incorporate two vortex points fixed at the poles of the sphere as an effect of rotation, and then investigate the evolution of coherent vortex structures. In this model, the effect of the Coriolis force is approximated just locally and no global interaction between the vortex points and the background flow is taken into consideration, which is a substantially different point from the real atmospheric flow. However, this is a simple model for the fluid motion on the sphere with the rotating effect, to which the analytic techniques for the two-dimensional flows are available. 
One of the coherent vortex structures is a vortex sheet, which is a discontinuous surface of the velocity field. Numerical study of the vortex sheet on the sphere with the pole vortices[18] indicates that it evolves into a structure with many rolling-up spirals whose centers are arranged equally along a line of latitude and that the number of the rolling-up spirals depends on the strengths of the pole vortices. In order to describe further long time evolution of the vortex sheet, by concentrating all the circulation contained in each of the spirals in its center point, we consider the motion of the vortex points that are equally spaced along the line of latitude. That is to say, let $\left(\Theta_{m}, \Psi_{m}\right)$ denote the position of the $m$ th vortex point in the spherical coordinates. Then the $N$-ring is represented by

$$
\Theta_{m}=\theta_{0}, \quad \Psi_{m}=\frac{2 \pi m}{N}, \quad m=1,2, \cdots, N .
$$

We call this polygonal configuration " $N$-ring". Generally speaking, since each of the spirals has different size, we need to consider the motion of $N$-ring with various strengths. However, for the sake of simplicity, the strengths of all the vortex points are assumed to be identical, say $\Gamma$, in the present paper.

The equations of the $N$-vortex points on the sphere with the pole vortices are given by

$$
\begin{aligned}
\dot{\Theta}_{m}= & -\frac{\Gamma}{4 \pi} \sum_{j \neq m}^{N} \frac{\sin \Theta_{j} \sin \left(\Psi_{m}-\Psi_{j}\right)}{1-\cos \gamma_{m j}}, \\
\sin \Theta_{m} \dot{\Psi}_{m}= & -\frac{\Gamma}{4 \pi} \sum_{j \neq m}^{N} \frac{\cos \Theta_{m} \sin \Theta_{j} \cos \left(\Psi_{m}-\Psi_{j}\right)-\sin \Theta_{m} \cos \Theta_{j}}{1-\cos \gamma_{m j}} \\
& +\frac{\Gamma_{1}}{4 \pi} \frac{\sin \Theta_{m}}{1-\cos \Theta_{m}}-\frac{\Gamma_{2}}{4 \pi} \frac{\sin \Theta_{m}}{1+\cos \Theta_{m}}, \quad m=1,2, \cdots, N,
\end{aligned}
$$

in which $\gamma_{m j}$ represents the central angle between the $m$ th and the $j$ th vortex points, and

$$
\cos \gamma_{m j}=\cos \Theta_{m} \cos \Theta_{j}+\sin \Theta_{m} \sin \Theta_{j} \cos \left(\Psi_{m}-\Psi_{j}\right) .
$$

The strengths of the north and the south pole vortices are denoted by $\Gamma_{1}$ and $\Gamma_{2}$ respectively. The equations define a Hamiltonian dynamical system in the phase space $\mathbb{P}_{N} \equiv[0, \pi]^{N} \times(\mathbb{R} / 2 \pi \mathbb{Z})^{N} \subset \mathbb{R}^{2 N}[8,14]$, whose Hamiltonian is given by

$$
\begin{aligned}
H= & -\frac{\Gamma^{2}}{8 \pi} \sum_{m=1}^{N} \sum_{j \neq m}^{N} \log \left(1-\cos \gamma_{m j}\right) \\
& -\frac{\Gamma_{1} \Gamma}{4 \pi} \sum_{m=1}^{N} \log \left(1-\cos \Theta_{m}\right)-\frac{\Gamma_{2} \Gamma}{4 \pi} \sum_{m=1}^{N} \log \left(1+\cos \Theta_{m}\right) .
\end{aligned}
$$

Note that the system has the invariant $\sum_{m=1}^{N} \cos \Theta_{m}$ due to the symmetry with respect to the rotation around the pole.

The stability of the $N$-ring on the sphere has been studied in many papers. Linear stability of the polygonal $N$-ring on the sphere was investigated by Polvani and Dritschel[16]. Then, Pekarsky and Marsden[15] studied the nonlinear stability of three vortices with arbitrary strengths, including the 3-ring, by the energy momentum method. Boatto and Cabral[2] carried out the linear and nonlinear stability analysis of the $N$-ring on the sphere in the sense of a sufficient condition due to Dirichlet. They showed that the range of the nonlinear stability coincided with that of the linear stability. Kurakin[9] recently considered the nonlinear stability in the sense of Routh and complemented the stability analysis of the $N$-ring on the sphere. 
The periodic orbits observed in the $N$-vortex points problem have become an important theme recently[11, 19, 21]. Souliére and Tokieda[21] and Laurent-Polz[11] gave algebraic methods to find periodic orbits and heteroclinic connections by reducing the system with its symmetry. In the paper [19], all the eigenvalues and their corresponding eigenvectors of the linearized equation for the stationary $N$-ring configuration were obtained, with which the even $N$-ring system was successfully reduced to an invariant two-dimensional integrable system. The reduced system showed the existence of the possible periodic orbits when the $N$-ring became unstable. In the present article, we generalize the techniques based on the linear stability analysis in order to understand the complicated evolution of the unstable odd $N$-ring.

Now, we review the results of the paper[19], which are required to describe our method. Substituting (1) into the equations (2) and (3), we obtain

$$
\dot{\Theta}_{m}=0, \quad \dot{\Psi}_{m}=V_{0}(N),
$$

in which

$$
V_{0}(N)=\frac{\Gamma_{1}-\Gamma_{2}}{4 \pi \sin ^{2} \theta_{0}}+\frac{\left(\Gamma_{1}+\Gamma_{2}+2 \pi\right) \cos \theta_{0}}{4 \pi \sin ^{2} \theta_{0}}-\frac{1}{2 N} \frac{\cos \theta_{0}}{\sin ^{2} \theta_{0}} .
$$

Hence, it is a steady solution in the spherical coordinates rotating in the longitudinal direction with the constant angular speed $V_{0}(N)$. When we perturb the steady solution,

$$
\Theta_{m}(t)=\theta_{0}+\epsilon \theta_{m}(t), \quad \Psi_{m}(t)=\frac{2 \pi m}{N}+V_{0}(N) t+\epsilon \varphi_{m}(t), \quad \epsilon \ll 1,
$$

then we have the linearized equations of $O(\epsilon)$ for the perturbations:

$$
\begin{aligned}
\dot{\theta}_{m} & =\frac{1}{2 N \sin \theta_{0}} \sum_{j \neq m}^{N} \frac{\varphi_{m}-\varphi_{j}}{1-\cos \frac{2 \pi}{N}(m-j)}, \\
\dot{\varphi}_{m} & =\frac{1}{2 N \sin ^{3} \theta_{0}} \sum_{j \neq m}^{N} \frac{\theta_{m}-\theta_{j}}{1-\cos \frac{2 \pi}{N}(m-j)}+B_{N} \theta_{m} .
\end{aligned}
$$

The parameter $B_{N}$ is represented by

$$
B_{N}=\frac{1+\cos ^{2} \theta_{0}}{2 N \sin ^{3} \theta_{0}}-\frac{\kappa_{1}\left(1+\cos ^{2} \theta_{0}\right)}{2 \sin ^{3} \theta_{0}}-\frac{\kappa_{2} \cos \theta_{0}}{2 \sin ^{3} \theta_{0}} .
$$

in which $\kappa_{1}$ and $\kappa_{2}$ are the equivalent parameters to $\Gamma_{1}$ and $\Gamma_{2}$ defined by

$$
\kappa_{1}=\frac{\Gamma_{1}+\Gamma_{2}+2 \pi}{2 \pi}, \quad \kappa_{2}=\frac{\Gamma_{1}-\Gamma_{2}}{\pi} .
$$

The eigenvalues of the linearized equations (6) and (7) are given explicitly as follows[19].

Theorem 1. For $p=0,1, \cdots, N-1$, the eigenvalues $\lambda_{p}^{ \pm}$are represented by

$$
\lambda_{p}^{ \pm}= \pm \sqrt{\xi_{p} \eta_{p}}
$$

in which

$$
\xi_{p}=\frac{p(N-p)}{2 N \sin \theta_{0}}, \quad \eta_{p}=\frac{p(N-p)}{2 N \sin ^{3} \theta_{0}}+B_{N}
$$


The expression (9) indicates that $\lambda_{0}^{ \pm}=0$ and $\lambda_{p}^{ \pm}=\lambda_{N-p}^{ \pm}$. Hence, when $N=$ $2 M$, there exist zero eigenvalues $\lambda_{0}^{ \pm}=0$, double eigenvalues $\lambda_{p}^{ \pm}$for $p=1, \cdots, M-1$, and simple eigenvalues $\lambda_{M}^{ \pm}$. When $N=2 M+1$, we have zero eigenvalues $\lambda_{0}^{ \pm}=0$ and double eigenvalues $\lambda_{p}^{ \pm}$for $p=1, \cdots, M$. Hence, the multiplicity of the eigenvalues $\lambda_{M}^{ \pm}$is different.

If $\xi_{p} \eta_{p}<0$, then $\lambda_{p}$ is pure imaginary due to (9). Therefore we have the following stability proposition.

Proposition 2. For $p=1,2, \cdots, M$, if $\eta_{p}<0$, or equivalently due to (10)

$$
\frac{p N-p^{2}}{N}+\frac{1+\cos ^{2} \theta_{0}}{N}<\kappa_{1}\left(1+\cos ^{2} \theta_{0}\right)+\kappa_{2} \cos \theta_{0} \equiv \kappa .
$$

then the eigenvalues $\lambda_{p}^{ \pm}$are pure imaginary numbers and consequently they are neutrally stable.

Note that the stability of the $N$-ring is determined by that of the largest eigenvalues, since the eigenvalues satisfy the following order due to (9),

$$
\left(\lambda_{1}^{ \pm}\right)^{2}<\left(\lambda_{2}^{ \pm}\right)^{2}<\cdots<\left(\lambda_{M}^{ \pm}\right)^{2}
$$

Thus, it is the double eigenvalue $\lambda_{M}^{+}$that becomes unstable when the odd $N$-ring loses its stability. Hence, the unstable manifold corresponding to $\lambda_{M}^{+}$has the twodimensional tangent space, and thus the motion of the perturbed $N$-ring could be complicated for the odd case. Actually, numerical computation of the 3-ring at the equator[20] pointed out the existence of a heteroclinic structure in the highdimensional phase space, which results in a non-trivial recurrent evolution. In the present paper, we confirm the numerical conjecture by a proposing projection method.

This paper consists of five sections. In $\S 2$, we introduce a Hamiltonian projection method, which makes it possible to project the iso-surface of the Hamiltonian on two-dimensional eigenspaces. Applying the projection method to the 3-ring and the 5-ring problems in $\S 3$ and $\S 4$ respectively, we investigate the complex evolution from the structure of the iso-surface of the Hamiltonian. Summary and remarks are given in the last section.

\section{Hamiltonian projection method}

Our projection method starts with the explicit representations of the eigenvectors corresponding to $\lambda_{p}^{ \pm}$, whose proof was given in [19].

Theorem 3. The eigenvectors $\vec{\phi}_{p}^{ \pm}$and $\vec{\psi}_{p}^{ \pm}$corresponding to the eigenvalues $\lambda_{p}^{ \pm}$for $p=0, \cdots, M$ are given by

$$
\begin{gathered}
\vec{\psi}_{p}^{ \pm}=t\left(\sqrt{\xi_{p}}, \sqrt{\xi_{p}} \cos \frac{2 \pi}{N} p, \cdots, \sqrt{\xi_{p}} \cos \frac{2 \pi}{N}(N-1) p,\right. \\
\left. \pm \sqrt{\eta_{p}}, \pm \sqrt{\eta_{p}} \cos \frac{2 \pi}{N} p, \cdots, \pm \sqrt{\eta_{p}} \cos \frac{2 \pi}{N}(N-1) p\right), \\
\vec{\phi}_{p}^{ \pm}=t\left(0, \sqrt{\xi_{p}} \sin \frac{2 \pi}{N} p, \cdots, \sqrt{\xi_{p}} \sin \frac{2 \pi}{N}(N-1) p,\right. \\
\left.0, \pm \sqrt{\eta_{p}} \sin \frac{2 \pi}{N} p, \cdots, \pm \sqrt{\eta_{p}} \sin \frac{2 \pi}{N}(N-1) p\right) .
\end{gathered}
$$


Noting that the eigenvectors have pure imaginary components if the eigenvalues $\lambda_{p}^{ \pm}$are neutrally stable, namely $\eta_{p}<0$, we define the inner product of the two vectors $\vec{x}={ }^{t}\left(x_{1}, x_{2}, \ldots, x_{N}\right)$ and $\vec{y}={ }^{t}\left(y_{1}, y_{2}, \ldots, y_{N}\right)$ by

$$
(\vec{x}, \vec{y}) \equiv \sum_{m=1}^{N} x_{i} y_{i}^{*},
$$

in which $y^{*}$ denotes the complex conjugate of $y$. Then, we have the following lemma.

Lemma 4. The eigenvectors $\vec{\psi}_{p}^{ \pm}$and $\vec{\phi}_{p}^{ \pm}$satisfy

$$
\begin{gathered}
\left(\vec{\psi}_{p}^{ \pm}, \vec{\phi}_{q}^{ \pm}\right)=0, \quad\left(\vec{\psi}_{p}^{ \pm}, \vec{\psi}_{q}^{ \pm}\right)=\left(\vec{\phi}_{p}^{ \pm}, \vec{\phi}_{q}^{ \pm}\right)=0 \text { for } p \neq q \\
\left(\vec{\psi}_{p}^{+}, \vec{\psi}_{p}^{-}\right)=\left(\vec{\phi}_{p}^{+}, \vec{\phi}_{p}^{-}\right)=\frac{N}{2}\left(\xi_{p}-\left|\eta_{p}\right|\right), \quad\left|\vec{\psi}_{p}^{ \pm}\right|^{2}=\left|\vec{\phi}_{p}^{ \pm}\right|^{2}=\frac{N}{2}\left(\xi_{p}+\left|\eta_{p}\right|\right) .
\end{gathered}
$$

Proof: It follows from the formula $\sum_{m=0}^{N-1} \omega_{p}^{m}=\frac{1-\omega_{p}^{N}}{1-\omega_{p}}=0$ for $\omega_{p}=e^{\frac{2 \pi \mathrm{i}_{p}}{N}}$ that

$$
\sum_{m=0}^{N-1} \cos \frac{2 \pi m p}{N}=\sum_{m=1}^{N-1} \sin \frac{2 \pi m p}{N}=0, \quad p=1,2, \cdots, N-1 .
$$

Hence, we have

$$
\begin{aligned}
\left(\vec{\psi}_{p}^{+}, \vec{\phi}_{q}^{ \pm}\right) & =\left(\sqrt{\xi_{p} \xi_{q}} \pm \sqrt{\eta_{p}}{\sqrt{\eta_{q}}}^{*}\right) \sum_{m=1}^{N-1} \cos \frac{2 \pi m p}{N} \sin \frac{2 \pi m q}{N} \\
& =\frac{1}{2}\left(\sqrt{\xi_{p} \xi_{q}} \pm \sqrt{\eta_{p}}{\sqrt{\eta_{q}}}^{*}\right) \sum_{m=1}^{N-1}\left(\sin \frac{2 \pi m(p+q)}{N}-\sin \frac{2 \pi m(p-q)}{N}\right)=0 .
\end{aligned}
$$

and for $p \neq q$

$$
\begin{aligned}
\left(\vec{\psi}_{p}^{+}, \vec{\psi}_{q}^{ \pm}\right) & =\left(\sqrt{\xi_{p} \xi_{q}} \pm \sqrt{\eta_{p}}{\sqrt{\eta_{q}}}^{*}\right) \sum_{m=0}^{N-1} \cos \frac{2 \pi m p}{N} \cos \frac{2 \pi m q}{N} \\
& =\frac{1}{2}\left(\sqrt{\xi_{p} \xi_{q}} \pm \sqrt{\eta_{p}}{\sqrt{\eta_{q}}}^{*}\right) \sum_{m=0}^{N-1}\left(\cos \frac{2 \pi m(p+q)}{N}+\cos \frac{2 \pi m(p-q)}{N}\right)=0 .
\end{aligned}
$$

Similarly, we obtain

$$
\begin{aligned}
\left(\vec{\psi}_{p}^{+}, \vec{\psi}_{p}^{ \pm}\right) & =\left(\xi_{p} \pm\left|\eta_{p}\right|\right) \sum_{m=0}^{N-1} \cos ^{2} \frac{2 \pi m p}{N} \\
& =\frac{1}{2}\left(\xi_{p} \pm\left|\eta_{p}\right|\right) \sum_{m=0}^{N-1}\left(\cos \frac{4 \pi m p}{N}+1\right)=\frac{N}{2}\left(\xi_{p} \pm\left|\eta_{p}\right|\right)
\end{aligned}
$$

In the same way, $\left(\vec{\phi}_{p}^{+}, \vec{\phi}_{p}^{ \pm}\right)=\frac{N}{2}\left(\xi_{p} \pm\left|\eta_{p}\right|\right)$.

In what follows, we assume that the number of the vortex points is odd, say $N=2 M+1$. Then, since the eigenvectors $\vec{\psi}_{p}^{ \pm}$and $\vec{\phi}_{p}^{ \pm}$for $p=1, \cdots, M$ are linearly independent, they form the basis of the $4 M$-dimensional subspace of $\mathbb{R}^{2 N}$. A basic idea of the Hamiltonian projection method is to express the motion of the $N$-ring by the linear combination of the eigenvectors, and so two more linearly independent vectors are required, which are simply provided in the following lemma. The proof is straightforward in view of (15). 
Lemma 5. Let $\vec{\zeta}^{ \pm}$be defined by

$$
\vec{\zeta}^{ \pm}=\frac{1}{\sqrt{2 N}}^{t}(1,1, \cdots, 1, \pm 1, \pm 1, \cdots, \pm 1) .
$$

Then, they satisfy $\left(\vec{\psi}_{p}^{ \pm}, \vec{\zeta}^{ \pm}\right)=0,\left(\vec{\phi}_{p}^{ \pm}, \vec{\zeta}^{ \pm}\right)=0$ and $\left(\vec{\zeta}^{+}, \vec{\zeta}^{-}\right)=0$.

Now, let the position of the vortex points $\vec{x}=\left(\Theta_{1}, \Theta_{2}, \cdots, \Theta_{N}, \Psi_{1}, \Psi_{2}, \cdots, \Psi_{N}\right) \in$ $\mathbb{P}_{N}$ be represented by the following linear combination of the eigenvectors and the complementary vectors.

$$
\vec{x}=\vec{x}_{0}+\sum_{p=1}^{M}\left(a_{p} \vec{\psi}_{p}^{+}+b_{p} \vec{\psi}_{p}^{-}+c_{p} \vec{\phi}_{p}^{+}+d_{p} \vec{\phi}_{p}^{-}\right)+e \vec{\zeta}^{+}+f \vec{\zeta}^{-},
$$

in which $a_{p}, b_{p}, c_{p}, d_{p}, e, f \in \mathbb{C}$, and $\vec{x}_{0}$ denotes the odd $N$-ring equilibrium, which is represented by

$$
\vec{x}_{0}=(\underbrace{\theta_{0}, \theta_{0}, \cdots, \theta_{0}}_{N}, 0, \underbrace{\frac{2 \pi}{N}, \frac{4 \pi}{N}, \cdots, \frac{2 \pi M}{N}}_{M}, \underbrace{-\frac{2 \pi M}{N}, \cdots,-\frac{4 \pi}{N},-\frac{2 \pi}{N}}_{M}) .
$$

Here, we adjust the equilibrium configuration longitudinally so that $\Psi_{1}=0$ for the sake of analytic convenience. Then, it follows from Lemma 4 and Lemma 5 that the coefficients are represented by

$$
\begin{aligned}
a_{p} & =\frac{1}{2 N \xi_{p}}\left(\vec{x}-\vec{x}_{0}, \vec{\psi}_{p}^{+}+\vec{\psi}_{p}^{-}\right)+\frac{1}{2 N\left|\eta_{p}\right|}\left(\vec{x}-\vec{x}_{0}, \vec{\psi}_{p}^{+}-\vec{\psi}_{p}^{-}\right), \\
b_{p} & =\frac{1}{2 N \xi_{p}}\left(\vec{x}-\vec{x}_{0}, \vec{\psi}_{p}^{+}+\vec{\psi}_{p}^{-}\right)-\frac{1}{2 N\left|\eta_{p}\right|}\left(\vec{x}-\vec{x}_{0}, \vec{\psi}_{p}^{+}-\vec{\psi}_{p}^{-}\right), \\
c_{p} & =\frac{1}{2 N \xi_{p}}\left(\vec{x}-\vec{x}_{0}, \vec{\phi}_{p}^{+}+\vec{\phi}_{p}^{-}\right)+\frac{1}{2 N\left|\eta_{p}\right|}\left(\vec{x}-\vec{x}_{0}, \vec{\phi}_{p}^{+}-\vec{\phi}_{p}^{-}\right), \\
d_{p} & =\frac{1}{2 N \xi_{p}}\left(\vec{x}-\vec{x}_{0}, \vec{\phi}_{p}^{+}+\vec{\phi}_{p}^{-}\right)-\frac{1}{2 N\left|\eta_{p}\right|}\left(\vec{x}-\vec{x}_{0}, \vec{\phi}_{p}^{+}-\vec{\phi}_{p}^{-}\right), \\
e & =\left(\vec{x}-\vec{x}_{0}, \vec{\zeta}^{+}\right), \quad f=\left(\vec{x}-\vec{x}_{0}, \vec{\zeta}^{-}\right) .
\end{aligned}
$$

Therefore, the constraint conditions for the projection of the evolution of the vortex points on the two-dimensional phase spaces spanned by $\vec{\psi}_{p}^{ \pm}, \vec{\phi}_{p}^{ \pm}$and $\vec{\zeta}^{ \pm}$are equivalent to

$$
\begin{array}{llll}
\vec{\psi}_{p}^{ \pm}: & a_{q}=b_{q}=0(q \neq p), & c_{q}=d_{q}=0(\forall q), & e=f=0, \\
\vec{\phi}_{p}^{ \pm}: & a_{q}=b_{q}=0(\forall q), & c_{q}=d_{q}=0(q \neq p), & e=f=0, \\
\vec{\zeta}^{ \pm}: & a_{q}=b_{q}=0(\forall q), & c_{q}=d_{q}=0(\forall q) . &
\end{array}
$$

Substituting (13), (14) and (17) into (19), we rewrite the constraint conditions for the projection as follows.

Proposition 6. The motion of the odd $N$-ring projected on the two-dimensional phase space spanned by $\vec{\psi}_{p}^{ \pm}$satisfies the following constraint conditions;

$$
\begin{array}{lll}
\sum_{k=1}^{N} \Theta_{k} \cos \frac{2 \pi q}{N}(k-1)=0, & \sum_{k=1}^{N} \Psi_{k} \cos \frac{2 \pi q}{N}(k-1)=0, & (q \neq p), \\
\sum_{k=2}^{N} \Theta_{k} \sin \frac{2 \pi q}{N}(k-1)=0, & \sum_{k=2}^{N} \Psi_{k} \sin \frac{2 \pi q}{N}(k-1)=2 \sum_{k=1}^{M} \frac{2 \pi}{N} k \sin \frac{2 \pi q}{N} k, & (\forall q), \\
\sum_{k=1}^{N} \Theta_{k}=N \theta_{0}, & \sum_{k=1}^{N} \Psi_{k}=0 . &
\end{array}
$$


Proposition 7. The motion of the odd N-ring projected on the two-dimensional phase space spanned by $\vec{\phi}_{p}^{ \pm}$satisfies the following constraint conditions;

$$
\begin{array}{lll}
\sum_{k=1}^{N} \Theta_{k} \cos \frac{2 \pi q}{N}(k-1)=0, & \sum_{k=1}^{N} \Psi_{k} \cos \frac{2 \pi q}{N}(k-1)=0, & (\forall q), \\
\sum_{k=2}^{N} \Theta_{k} \sin \frac{2 \pi q}{N}(k-1)=0, & \sum_{k=2}^{N} \Psi_{k} \sin \frac{2 \pi q}{N}(k-1)=2 \sum_{k=1}^{M} \frac{2 \pi}{N} k \sin \frac{2 \pi q}{N} k, & (q \neq p), \\
\sum_{k=1}^{N} \Theta_{k}=N \theta_{0}, & \sum_{k=1}^{N} \Psi_{k}=0 .
\end{array}
$$

Proposition 8. The motion of the odd $N$-ring projected on the two-dimensional phase space spanned by $\vec{\zeta}^{ \pm}$satisfies the following constraint conditions; For $1 \leq q \leq$ $M$, they are

$$
\begin{array}{ll}
\sum_{k=1}^{N} \Theta_{k} \cos \frac{2 \pi q}{N}(k-1)=0, & \sum_{k=1}^{N} \Psi_{k} \cos \frac{2 \pi q}{N}(k-1)=0 \\
\sum_{k=2}^{N} \Theta_{k} \sin \frac{2 \pi q}{N}(k-1)=0, & \sum_{k=2}^{N} \Psi_{k} \sin \frac{2 \pi q}{N}(k-1)=2 \sum_{k=1}^{M} \frac{2 \pi}{N} k \sin \frac{2 \pi q}{N} k .
\end{array}
$$

Since the perturbed $N$-ring evolves in the iso-surface of the Hamiltonian embedded in $\mathbb{P}_{N}$, and hardly grows in the directions of the neutrally stable eigenvectors, we project the iso-surface of the Hamiltonian on the planar phase space spanned by the pair of the unstable and the stable eigenvectors with the above constraint conditions in order to observe the unstable evolution of the $N$-ring. Generally speaking, the projection conditions (20) and (21) just restrict the system to the eigenspaces, and fail to reduce the $N$-ring system to the two-dimensional invariant dynamical system. That is to say, the projected iso-surface of the Hamiltonian disagree with the actual orbit of the $N$-ring evolution. In addition, since the projection is based on the local expansion (16), it also provides us with a little information on the global structure of the iso-surface of the Hamiltonian. However, as we will see in the following sections, applying the projection method to the 3-ring and the 5-ring cases, we can describe their complicated evolutions when the largest eigenvalue $\lambda_{M}^{+}$ becomes unstable from the projected iso-surface. In particular, for a special 3ring system, the projection method successfully reduce the system to the invariant two-dimensional dynamical system, from which we can show that there exists a heteroclinic orbit in the high-dimensional phase space.

Finally, we mention the meaning of the projection of the iso-surface of the Hamiltonian on the special subspace spanned by $\vec{\zeta}^{ \pm}$. It is easy to rewrite the constraint conditions in Proposition 8 in a simpler form.

Corollary 9. The constraint conditions (22) are equivalent to

$$
\begin{gathered}
\Theta_{1}=\Theta_{2}=\cdots=\Theta_{N}, \\
\Psi_{j}=\left\{\begin{array}{l}
\Psi_{1}+\frac{2 \pi}{N}(j-1), \\
\Psi_{1}-\frac{2 \pi}{N}(N-j+1), \quad M+2 \leq j \leq N .
\end{array}\right.
\end{gathered}
$$

Proof: It follows from (15) that the condition (23) satisfies the equations for the $\Theta$-variables in (22). Next we can show that the condition (24) solves the equations 
for the $\Psi$-variables as follows:

$$
\begin{aligned}
\sum_{k=1}^{N} \Psi_{k} \cos \frac{2 \pi q}{N}(k-1)= & \sum_{k=1}^{M+1}\left(\Psi_{1}+\frac{2 \pi}{N}(k-1)\right) \cos \frac{2 \pi q}{N}(k-1) \\
& +\sum_{k=M+2}^{N}\left(\Psi_{1}-\frac{2 \pi}{N}(N-k+1)\right) \cos \frac{2 \pi q}{N}(k-1) \\
= & \Psi_{1} \sum_{k=1}^{N} \cos \frac{2 \pi q}{N}(k-1)+\sum_{k=2}^{M+1} \frac{2 \pi}{N}(k-1) \cos \frac{2 \pi q}{N}(k-1) \\
& -\sum_{k=M+2}^{N} \frac{2 \pi}{N}(N-k+1) \cos \frac{2 \pi q}{N}(k-1)
\end{aligned}
$$

The first term becomes zero due to (15).In the last term, exchanging the variables $k$ to $k^{\prime}$ by $N-k+1=k^{\prime}-1$, we have

$$
\begin{aligned}
& \sum_{k=2}^{M+1} \frac{2 \pi}{N}(k-1) \cos \frac{2 \pi q}{N}(k-1)-\sum_{k^{\prime}=2}^{M+1} \frac{2 \pi}{N}\left(k^{\prime}-1\right) \cos \frac{2 \pi q}{N}\left(N-k^{\prime}+1\right) \\
= & \sum_{k=2}^{M+1} \frac{2 \pi}{N}(k-1) \cos \frac{2 \pi q}{N}(k-1)-\sum_{k^{\prime}=2}^{M+1} \frac{2 \pi}{N}\left(k^{\prime}-1\right) \cos \frac{2 \pi q}{N}\left(k^{\prime}-1\right)=0 .
\end{aligned}
$$

In the similar way, we obtain

$$
\begin{aligned}
\sum_{k=1}^{N} \Psi_{k} \sin \frac{2 \pi q}{N}(k-1)= & \sum_{k=2}^{M+1} \frac{2 \pi}{N}(k-1) \sin \frac{2 \pi q}{N}(k-1) \\
& -\sum_{k^{\prime}=2}^{M+1} \frac{2 \pi}{N}\left(k^{\prime}-1\right) \sin \frac{2 \pi q}{N}\left(N-k^{\prime}+1\right) \\
= & 2 \sum_{k=2}^{M+1} \frac{2 \pi}{N}(k-1) \sin \frac{2 \pi q}{N}(k-1) \\
= & 2 \sum_{k=1}^{M} \frac{2 \pi}{N} k \sin \frac{2 \pi q}{N} k
\end{aligned}
$$

Imposing the constraint conditions (23) on the Hamiltonian (4), we have the projected Hamiltonian $H_{\vec{\zeta}^{ \pm}}$:

$$
\begin{aligned}
H_{\vec{\zeta}^{ \pm}}= & -\frac{\Gamma^{2}}{8 \pi} \sum_{m=1}^{N} \sum_{j \neq m}^{N} \log \left(1-\cos ^{2} \Theta_{1}-\sin ^{2} \Theta_{1} \cos \left(\Psi_{m}-\Psi_{j}\right)\right) \\
& -\frac{\Gamma_{1} \Gamma}{4 \pi} N \log \left(1-\cos \Theta_{1}\right)-\frac{\Gamma_{2} \Gamma}{4 \pi} N \log \left(1+\cos \Theta_{1}\right) .
\end{aligned}
$$

Since (24) indicates that $\Psi_{m}-\Psi_{j}$ is independent of $\Psi_{1}$, the projected Hamiltonian depends only on the variables $\Theta_{1}$. Hence, the contour of the projected Hamiltonian in the phase space $\left(\Theta_{1}, \Psi_{1}\right)$ is equivalent to a straight line, $\Theta_{1}=$ Constant. In addition, the constraint conditions (23) and (24) indicate that when the orbit of the vortex points intersects the $\vec{\zeta}^{ \pm}$-plane, the vortex points form the $N$-ring configuration. In other words, if the distance between the orbit and the $\vec{\zeta}^{ \pm}$-plane, defined 
by

$$
\begin{aligned}
L_{\zeta}^{(N)} & =\left|\vec{x}-\vec{x}_{0}-e \vec{\zeta}^{+}-f \vec{\zeta}^{-}\right| \\
& =\sqrt{\frac{N}{2} \sum_{p=1}^{M}\left\{\xi_{p}\left(\left|a_{p}+b_{p}^{*}\right|^{2}+\left|c_{p}+d_{p}^{*}\right|^{2}\right)+\left|\eta_{p}\right|\left(\left|a_{p}-b_{p}^{*}\right|^{2}+\left|c_{p}-d_{p}^{*}\right|^{2}\right)\right\}}
\end{aligned}
$$

vanishes, the configuration of the vortex points becomes the $N$-ring.

\section{Heteroclinic connections in the 3-ring}

Then $N=3$, the constraint conditions in Proposition 6 and Proposition 7 are equivalent to

$$
\vec{\psi}_{1}^{ \pm}:\left\{\begin{array}{ll}
\Theta_{2}-\Theta_{3} & =0, \\
\Theta_{1}+\Theta_{2}+\Theta_{3} & =3 \theta_{0}, \\
\Psi_{2}-\Psi_{3} & =\frac{4}{3} \pi, \\
\Psi_{1}+\Psi_{2}+\Psi_{3} & =0,
\end{array} \quad \vec{\phi}_{1}^{ \pm}:\left\{\begin{array}{l}
2 \Theta_{1}-\Theta_{2}-\Theta_{3}=0, \\
\Theta_{1}+\Theta_{2}+\Theta_{3}=3 \theta_{0}, \\
2 \Psi_{1}-\Psi_{2}-\Psi_{3}=0 \\
\Psi_{1}+\Psi_{2}+\Psi_{3}=0 .
\end{array}\right.\right.
$$

Solving the equations, we have the relations between the variables as follows.

$$
\vec{\psi}_{1}^{ \pm}:\left\{\begin{array}{ll}
\Theta_{1}=3 \theta_{0}-2 \Theta_{2}, & \Theta_{3}=\Theta_{2}, \\
\Psi_{1}=-2 \Psi_{2}+\frac{4}{3} \pi, & \Psi_{3}=\Psi_{2}-\frac{4}{3} \pi,
\end{array} \vec{\phi}_{1}^{ \pm}: \begin{cases}\Theta_{1}=\theta_{0}, & \Theta_{3}=2 \theta_{0}-\Theta_{2}, \\
\Psi_{1}=0, & \Psi_{3}=-\Psi_{2} .\end{cases}\right.
$$

Substituting these relations into (4), we have the following projected Hamiltonians;

$$
\begin{aligned}
H_{\vec{\psi}_{1}^{ \pm}}= & -\frac{\Gamma^{2}}{4 \pi} \log \left(1-\cos \left(3 \theta_{0}-2 \Theta_{2}\right) \cos \Theta_{2}-\sin \left(3 \theta_{0}-2 \Theta_{2}\right) \sin \Theta_{2} \cos \left(3 \Psi_{2}-\frac{4}{3} \pi\right)\right) \\
& -\frac{\Gamma^{2}}{4 \pi} \log \left(1-\cos \left(3 \theta_{0}-2 \Theta_{2}\right) \cos \Theta_{2}-\sin \left(3 \theta_{0}-2 \Theta_{2}\right) \sin \Theta_{2} \cos \left(3 \Psi_{2}-\frac{2}{3} \pi\right)\right) \\
& -\frac{\Gamma^{2}}{4 \pi} \log \left(1-\cos ^{2} \Theta_{2}+\frac{1}{2} \sin ^{2} \Theta_{2}\right) \\
& -\frac{\Gamma_{1} \Gamma}{4 \pi} \log \left(1-\cos \left(3 \theta_{0}-2 \Theta_{2}\right)\right)\left(1-\cos \Theta_{2}\right)^{2} \\
& -\frac{\Gamma_{2} \Gamma}{4 \pi} \log \left(1+\cos \left(3 \theta_{0}-2 \Theta_{2}\right)\right)\left(1+\cos \Theta_{2}\right)^{2},
\end{aligned}
$$

and

$$
\begin{aligned}
H_{\vec{\phi}_{1}^{ \pm}}= & -\frac{\Gamma^{2}}{4 \pi} \log \left(1-\cos \theta_{0} \cos \Theta_{2}-\sin \theta_{0} \sin \Theta_{2} \cos \Psi_{2}\right) \\
& -\frac{\Gamma^{2}}{4 \pi} \log \left(1-\cos \theta_{0} \cos \left(2 \theta_{0}-\Theta_{2}\right)-\sin \theta_{0} \sin \left(2 \theta_{0}-\Theta_{2}\right) \cos \Psi_{2}\right) \\
& -\frac{\Gamma^{2}}{4 \pi} \log \left(1-\cos \Theta_{2} \cos \left(2 \theta_{0}-\Theta_{2}\right)-\sin \Theta_{2} \sin \left(2 \theta_{0}-\Theta_{2}\right) \cos 2 \Psi_{2}\right) \\
& -\frac{\Gamma_{1} \Gamma}{4 \pi} \log \left(1-\cos \theta_{0}\right)\left(1-\cos \Theta_{2}\right)\left(1-\cos \left(2 \theta_{0}-\Theta_{2}\right)\right) \\
& -\frac{\Gamma_{2} \Gamma}{4 \pi} \log \left(1+\cos \theta_{0}\right)\left(1+\cos \Theta_{2}\right)\left(1+\cos \left(2 \theta_{0}-\Theta_{2}\right)\right) .
\end{aligned}
$$

We plot the contour lines of $H_{\vec{\psi}_{1}^{ \pm}}$and $H_{\vec{\phi}_{1}^{ \pm}}$in the $\left(\Psi_{2}, \Theta_{2}\right)$-plane to see the global structure of the iso-surface of the Hamiltonian. When plotting the contours, we 
must note that the variable $\Theta_{2}$ moves in the range of $[0, \pi] \cap\left[\frac{1}{2}\left(3 \theta_{0}-\pi\right), \frac{3}{2} \theta_{0}\right]$ for $H_{\vec{\psi}_{1}^{ \pm}}$, and $[0, \pi] \cap\left[2 \theta_{0}-\pi, 2 \theta_{0}\right]$ for $H_{\vec{\phi}_{1}^{ \pm}}$respectively, due to $0 \leq \Theta_{1}, \Theta_{2}, \Theta_{3} \leq \pi$.

According to Proposition 2, the stability of the largest eigenvalues $\lambda_{1}^{ \pm}$changes at $\kappa=1+\frac{1}{3} \cos ^{2} \theta_{0}$. Hence, we show the contour plot of the projected Hamiltonians for the unstable case, $\kappa<1+\frac{1}{3} \cos ^{2} \theta_{0}$. Figure 1 (a) and (b) show the contour plots of $H_{\vec{\phi}_{1}^{ \pm}}$and $H_{\vec{\psi}_{1}^{ \pm}}$respectively, when $\left(\Gamma_{1}, \Gamma_{2}\right)=(-0.2 \pi,-0.2 \pi)$ and the 3-ring is located at the equator, i.e. $\theta_{0}=\frac{\pi}{2}$. In Figure 1 (a), the restricted iso-surface departing from $\left(\Psi_{2}, \Theta_{2}\right)=\left(\frac{2}{3} \pi, \frac{\pi}{2}\right)$, which corresponds to the original 3-ring configuration $\left(\frac{\pi}{2}, \frac{\pi}{2}, \frac{\pi}{2}, 0, \frac{2}{3} \pi,-\frac{2}{3} \pi\right) \in \mathbb{P}_{3}$, connects to another 3-ring equilibrium $\left(\Psi_{2}, \Theta_{2}\right)=\left(\frac{4}{3} \pi, \frac{\pi}{2}\right)$, which is the mirror symmetric 3-ring equilibrium $\left(\frac{\pi}{2}, \frac{\pi}{2}, \frac{\pi}{2}, 0,-\frac{2}{3} \pi, \frac{2}{3} \pi\right)$. As a matter of fact, when $\Gamma_{n}=\Gamma_{s}$ and $\theta_{0}=\frac{\pi}{2}$, the projection condition to the $\vec{\phi}_{1}^{ \pm}$-plane reduces the 3 -ring system to the invariant two-dimensional system embedded in the high-dimensional phase space, since substitution of the condition to the original equations yields $\dot{\Theta}_{1}=0, \dot{\Theta}_{2}+\dot{\Theta}_{3}=0, \dot{\Psi}_{1}=0$ and $\dot{\Psi}_{2}+\dot{\Psi}_{3}=0$. Hence, the contour line in Figure 1 (a) agrees with the actual orbit of the three vortex points, which indicates that there really exists the heteroclinic orbit connecting between the 3-ring and the mirror symmetric 3-ring. On the other hand, while the projection condition to the $\vec{\psi}_{1}^{ \pm}$-plane does not reduce the dynamical system, the restricted iso-surface in Figure 1 (b) has the heteroclinic connection between the 3 -ring and the longitudinally shifted 3 -ring equilibrium, $\left(\frac{\pi}{2}, \frac{\pi}{2}, \frac{\pi}{2}, \frac{2}{3} \pi,-\frac{2}{3} \pi, 0\right) \in \mathbb{P}_{3}$. Then, when the unstable 3 -ring is slightly perturbed, the orbit of the perturbed 3 -ring evolves in an iso-surface of the Hamiltonian in the neighborhood of the heteroclinic structure. We confirm the invariant two-dimensional dynamical system and the heteroclinic connection of the iso-surface affect the evolution of the unstable $N$-ring.

In order to observe the evolution of the perturbed 3-ring configuration quantitatively, we use the distance $L_{\zeta}^{(3)}$. Since $\eta_{1}>0$ when the 3-ring is linearly unstable due to Proposition 2, all the coefficients $a_{1}, b_{1}, c_{1}$ and $d_{1}$ are real due to (13), (14) and (18). Hence, the distance $L_{\zeta}^{(3)}$ is explicitly given by

$$
\begin{aligned}
L_{\zeta}^{(3)} & =\sqrt{\frac{3}{2} \xi_{1}\left(\left(a_{1}+b_{1}\right)^{2}+\left(c_{1}+d_{1}\right)^{2}\right)+\frac{3}{2} \eta_{1}\left(\left(a_{1}-b_{1}\right)^{2}+\left(c_{1}-d_{1}\right)^{2}\right)} \\
& =\sqrt{\frac{\left(2 \Theta_{1}-\Theta_{2}-\Theta_{3}\right)^{2}}{6}+\frac{\left(\Theta_{2}-\Theta_{3}\right)^{2}}{2}+\frac{\left(2 \Psi_{1}-\Psi_{2}-\Psi_{3}\right)^{2}}{6}+\frac{\left(\Psi_{2}-\Psi_{3}-\frac{4}{3} \pi\right)^{2}}{2}} .
\end{aligned}
$$

Here, instead of $L_{\zeta}^{(3)}$, we introduce the following distance $l_{\zeta}^{(3)}$ :

$l_{\zeta}^{(3)}=\sqrt{\frac{\left(2 \Theta_{1}-\Theta_{2}-\Theta_{3}\right)^{2}}{6}+\frac{\left(\Theta_{2}-\Theta_{3}\right)^{2}}{2}+\frac{1}{6} \sin ^{2} \frac{2 \Psi_{1}-\Psi_{2}-\Psi_{3}}{4}+\frac{1}{2} \sin ^{2} \frac{\Psi_{2}-\Psi_{3}-\frac{4}{3} \pi}{4} .}$

These two distances are the same in the sense that they measure the distance between the orbit of the three vortex points and the $\zeta^{ \pm}$-plane in $\mathbb{P}_{3}$. That is to say, if $l_{\zeta}^{(3)}=0$, then $2 \Theta_{1}-\Theta_{2}-\Theta_{3}=\Theta_{2}-\Theta_{3}=0,2 \Psi_{1}-\Psi_{2}-\Psi_{3}=4 n \pi$ and $\Psi_{2}-\Psi_{3}-\frac{4}{3} \pi=4 m \pi$ for $m, n \in \mathbb{Z}$, which leads to $L_{\zeta}^{(3)}=0$ because $\Psi_{i}$ is $2 \pi$-periodic. Hence when $l_{\zeta}^{(3)}=0$, then the three vortex points form the 3 ring configuration. On the other hand, when the vortex points form the mirror symmetric 3 -ring configuration, we have $l_{\zeta}^{(3)}=\frac{\sqrt{6}}{4}$. Therefore, if $l_{\zeta}^{(3)}=0$ or $\frac{\sqrt{6}}{4}$, the three vortex points form the 3-ring configuration.

The initial configuration of the three vortex points are given by

$$
\left(\Theta_{1}(0), \Theta_{2}(0), \Theta_{3}(0), \Psi_{1}(0), \Psi_{2}(0), \Psi_{3}(0)\right)=\vec{x}_{0}+\epsilon\left(\mu \vec{\psi}_{1}^{+}+(1-\mu) \vec{\phi}_{1}^{+}\right),
$$


in which $\epsilon=10^{-7}$ is the small amplitude of perturbation. The parameter $\mu(0 \leq$ $\mu \leq 1$ ) denotes the ratio of the initial unstable direction, since the unstable manifold is tangent to the plane spanned by $\vec{\phi}_{1}^{+}$and $\vec{\psi}_{1}^{+}$.

We show the evolutions of the perturbed 3-ring for various $\mu$. First, Figure 2 (a) shows the evolutions of $\cos \Theta_{1}, \cos \Theta_{2}$ and $\cos \Theta_{3}$ when the initial unstable direction is $\mu=0.0$. Since the initial perturbation lies in the neighborhood of the two-dimensional invariant dynamical system the $\vec{\phi}_{1}^{ \pm}$-plane, the evolution follows the heteroclinic orbit in the two-dimensional reduced system at the initial time interval. Indeed, the distance $l_{\zeta}^{(3)}$ shows that it stays zero up to around $t=70$ and then transfers to $\frac{\sqrt{6}}{4}$ at $t=100$, which indicates that the 3 -ring transforms to the mirror symmetric 3-ring configuration. After then, due to the existence of the unstable direction $\vec{\psi}_{1}^{+}$transverse to the reduced system, the orbit eventually goes off the reduced phase space and exhibits the complicated behavior. However, the evolution repeat not periodic but similar pattern, in which the evolution sometimes acquires the symmetry $\cos \Theta_{1}=0$ and $\cos \Theta_{2}+\cos \Theta_{3}=0$, in other words $\Theta_{1}=\frac{\pi}{2}$ and $\Theta_{2}+\Theta_{3}=\pi$, at around $t=400$ and $t=550$. At $t=400$, the evolution approaches the reduced invariant dynamical system in the $\vec{\phi}_{1}^{ \pm}$-plane and follows in the neighborhood of the heteroclinic orbit, since $l_{\zeta}^{(3)}$ moves near zero to $\frac{\sqrt{6}}{4}$. At $t=550$, the distance is just approaching the mirror symmetric 3 -ring equilibrium.

Second, we plot in Figure 3 the evolutions of (a) $\cos \Theta_{1}, \cos \Theta_{2}$ and $\cos \Theta_{3}$, and (b) the distances $l_{\zeta}^{(3)}$ for $\mu=1$.0. Although the direction of the perturbation $\vec{\psi}_{1}^{ \pm}$ is transverse to the $\vec{\phi}_{1}^{ \pm}$-plane, the evolution repeats similar pattern like $\mu=0.0$. The orbit approaches in the neighborhood of the hetercolinic orbit in the reduced dynamical system. Actually, the distance $l_{\zeta}^{(3)}$ transfers from near $\frac{\sqrt{6}}{4}$ to zero at around $t=280$. These two examples indicates that there exists an orbit of the perturbed 3-ring returning to the invariant dynamical system.

Third, we plot the evolutions of $\cos \Theta_{1}, \cos \Theta_{2}$ and $\cos \Theta_{3}$ in Figure 4. They show periodic behavior and enter in the vicinity of the zero-line periodically, at which the three vortices forms the 3 -ring equilibrium at the equator. The periodic evolution occurs when the orbit evolves in the closed iso-surfaces inside the heteroclinic connections in the restricted $\vec{\psi}_{1}^{ \pm}$and the reduced $\vec{\phi}_{1}^{ \pm}$-planes. The evolution of the distance $l_{\zeta}^{(3)}$ in Figure 4 (b) shows that it approaches zero when both $\cos \Theta_{1}$ and $\cos \Theta_{2}$ get into the neighborhood of the zero line. Hence, the three vortex points return to the original 3-ring configuration periodically.

The above three examples support the heteroclinic connections affect the evolution of the unstable 3-ring at the equator. On the other hand, however, since the orbit of the perturbed 3-ring is just a one-dimensional curve evolving in the four dimensional iso-surface of the Hamiltonian, it does not always follow the heteroclinic orbit nor return in the neighborhood of the invariant dynamical system. Actually, as we see in Figure 5 showing (a) the evolutions of $\cos \Theta_{1}, \cos \Theta_{2}$ and $\cos \Theta_{3}$ and (b) the distance for $\mu=0.7$, while the distance occasionally approaches near zero or $\frac{\sqrt{6}}{4}$, the evolutions look very irregular. Thus, the evolutions of the perturbed 3-ring also depends on the initial distance of the perturbation.

The heteroclinic connection of the iso-surface of the Hamiltonian is still observed when the 3-ring is located at another latitude. Figure 6 shows the contour plots of (a) $H_{\vec{\phi}_{1}^{ \pm}}$and (b) $H_{\vec{\psi}_{1}^{ \pm}}$, and (c) the evolution of the distance $l_{\zeta}^{(3)}$ with the initial direction $\mu=0.2$ when the 3 -ring is located at $\theta_{0}=\frac{\pi}{4}$ and the strengths of the pole vortices are given by $\left(\Gamma_{1}, \Gamma_{2}\right)=(-0.3 \pi,-0.4 \pi)$. Since the projection condition to the $\vec{\phi}_{1}^{ \pm}$-plane no longer reduces the system to the two-dimensional invariant dynamical system, we conclude nothing about the existence of the heteroclinic orbit like the previous case. However, since both of the restricted iso-surfaces of the Hamiltonian departing from the 3-ring in Figure 6 (a) and (b) have the heteroclinic connections 
(a)

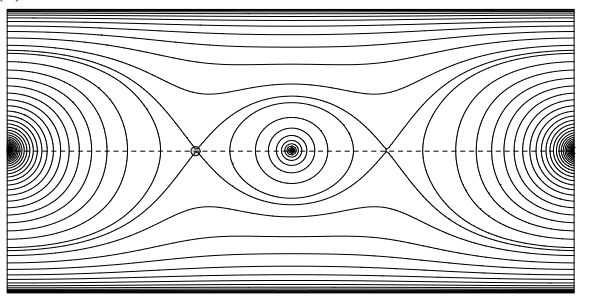

(b)

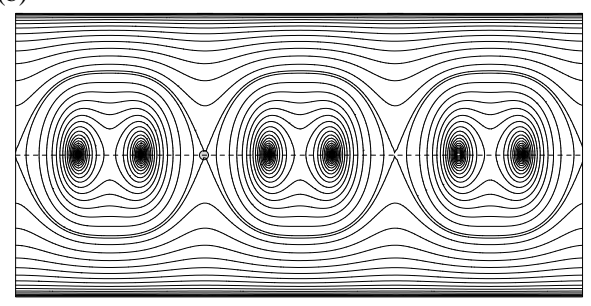

Figure 1: Contour plots of the projected Hamiltonians (a) $H_{\vec{\phi}_{1}^{ \pm}}$and (b) $H_{\vec{\psi}_{1}^{ \pm}}$ in $\left(\Psi_{2}, \Theta_{2}\right)$-plane, when the 3 -ring is located at the equator for $\left(\Gamma_{1}, \Gamma_{2}\right)=$ $(-0.2 \pi,-0.2 \pi)$. The circle in the figures denotes the steady 3 -ring.

with the mirror symmetric 3-ring and the longitudinally shifted 3-ring respectively, the orbit of the perturbed 3-ring is governed by the heteroclinic structure. Indeed, the distance $l_{\zeta}^{(3)}$ for the evolution of the perturbed 3-ring for $\mu=0.2$ in Figure 6 (c) shows that it evolves regularly with returning to the 3-ring configuration.

\section{Homocllinic connections in the 5-ring}

When $N=5$, solving the constraint conditions in Proposition 6 and 7, we have the following relations between the variables:

$$
\vec{\psi}_{2}^{ \pm}:\left\{\begin{array}{l}
\Theta_{1}=\sqrt{5} \theta_{0}+(1-\sqrt{5}) \Theta_{2}, \\
\Theta_{3}=\frac{1}{2}(5-\sqrt{5}) \theta_{0}-\frac{1}{2}(3-\sqrt{5}) \Theta_{2}, \\
\Theta_{4}=\frac{1}{2}(5-\sqrt{5}) \theta_{0}-\frac{1}{2}(3-\sqrt{5}) \Theta_{2}, \\
\Theta_{5}=\Theta_{2}, \\
\Psi_{1}=(1-\sqrt{5})\left(\Psi_{2}-\frac{2}{5} \pi\right), \\
\Psi_{3}=\frac{4}{5} \pi-\frac{1}{2}(3-\sqrt{5})\left(\Psi_{2}-\frac{2}{5} \pi\right), \\
\Psi_{4}=-\frac{4}{5} \pi-\frac{1}{2}(3-\sqrt{5})\left(\Psi_{2}-\frac{2}{5} \pi\right), \\
\Psi_{5}=\Psi_{2}-\frac{4}{5} \pi,
\end{array} \quad \vec{\psi}_{1}^{ \pm}: \quad\left\{\begin{array}{l}
\Theta_{1}=-\sqrt{5} \theta_{0}+(1-\sqrt{5}) \Theta_{2}, \\
\Theta_{3}=\frac{1}{2}(5+\sqrt{5}) \theta_{0}-\frac{1}{2}(3+\sqrt{5}) \Theta_{2}, \\
\Theta_{4}=\frac{1}{2}(5+\sqrt{5}) \theta_{0}-\frac{1}{2}(3+\sqrt{5}) \Theta_{2}, \\
\Theta_{5}=\Theta_{2}, \\
\Psi_{1}=(1+\sqrt{5})\left(\Psi_{2}-\frac{2}{5} \pi\right), \\
\Psi_{3}=\frac{4}{5} \pi-\frac{1}{2}(3+\sqrt{5})\left(\Psi_{2}-\frac{2}{5} \pi\right), \\
\Psi_{4}=-\frac{4}{5} \pi-\frac{1}{2}(3+\sqrt{5})\left(\Psi_{2}-\frac{2}{5} \pi\right), \\
\Psi_{5}=\Psi_{2}-\frac{4}{5} \pi,
\end{array}\right.\right.
$$

and

$$
\vec{\phi}_{2}^{ \pm}:\left\{\begin{array}{l}
\Theta_{1}=\theta_{0}, \\
\Theta_{3}=\theta_{0}-\frac{1}{2}(\sqrt{5}+1)\left(\Theta_{2}-\theta_{0}\right), \\
\Theta_{4}=\theta_{0}+\frac{1}{2}(\sqrt{5}+1)\left(\Theta_{2}-\theta_{0}\right), \\
\Theta_{5}=2 \theta_{0}-\Theta_{2}, \\
\Psi_{1}=0, \\
\Psi_{3}=\frac{4}{5} \pi-\frac{1}{2}(\sqrt{5}+1)\left(\Psi_{2}-\frac{2}{5} \pi\right), \\
\Psi_{4}=-\frac{4}{5} \pi+\frac{1}{2}(\sqrt{5}+1)\left(\Psi_{2}-\frac{2}{5} \pi\right), \\
\Psi_{5}=-\Psi_{2},
\end{array} \quad \vec{\phi}_{1}^{ \pm}: \quad\left\{\begin{array}{l}
\Theta_{1}=\theta_{0}, \\
\Theta_{3}=\theta_{0}+\frac{1}{2}(\sqrt{5}-1)\left(\Theta_{2}-\theta_{0}\right), \\
\Theta_{4}=\theta_{0}-\frac{1}{2}(\sqrt{5}-1)\left(\Theta_{2}-\theta_{0}\right), \\
\Theta_{5}=2 \theta_{0}-\Theta_{2}, \\
\Psi_{1}=0, \\
\Psi_{3}=\frac{4}{5} \pi+\frac{1}{2}(\sqrt{5}-1)\left(\Psi_{2}-\frac{2}{5} \pi\right), \\
\Psi_{4}=-\frac{4}{5} \pi-\frac{1}{2}(\sqrt{5}-1)\left(\Psi_{2}-\frac{2}{5} \pi\right), \\
\Psi_{5}=-\Psi_{2} .
\end{array}\right.\right.
$$

Substituting them into the Hamiltonian (4), we obtain the projected Hamiltonians $H_{\vec{\psi}_{2}^{ \pm}}, H_{\vec{\psi}_{1}^{ \pm}}, H_{\vec{\phi}_{2}^{ \pm}}$and $H_{\vec{\phi}_{1}^{ \pm}}$. Linear stability analysis for $N=5$ indicates that the largest eigenvalue $\lambda_{2}^{+}$becomes unstable at $\kappa=\frac{1}{5}\left(7+\cos ^{2} \theta_{0}\right)$ and $\lambda_{1}^{+}$becomes unstable at $\kappa=\frac{1}{5}\left(5+\cos ^{2} \theta_{0}\right)$. Thus we plot the contour lines of the projected Hamiltonians $H_{\vec{\psi}_{2}^{ \pm}}$and $H_{\vec{\phi}_{2}^{ \pm}}$in $\left(\Theta_{2}, \Psi_{2}\right)$-plane when only the largest eigenvalue $\lambda_{2}^{+}$ is unstable, namely $\frac{1}{5}\left(5+\cos ^{2} \theta_{0}\right)<\kappa<\frac{1}{5}\left(7+\cos ^{2} \theta_{0}\right)$. On the other hand, when both of the eigenvalues $\lambda_{2}^{+}$and $\lambda_{1}^{+}$are unstable, i.e. $\frac{1}{5}\left(5+\cos ^{2} \theta_{0}\right)>\kappa$, we plot the contour lines of $H_{\vec{\psi}_{2}^{ \pm}}, H_{\vec{\phi}_{2}^{ \pm}}, H_{\vec{\psi}_{1}^{ \pm}}$and $H_{\vec{\phi}_{1}^{ \pm}}$. We note again that we have to 

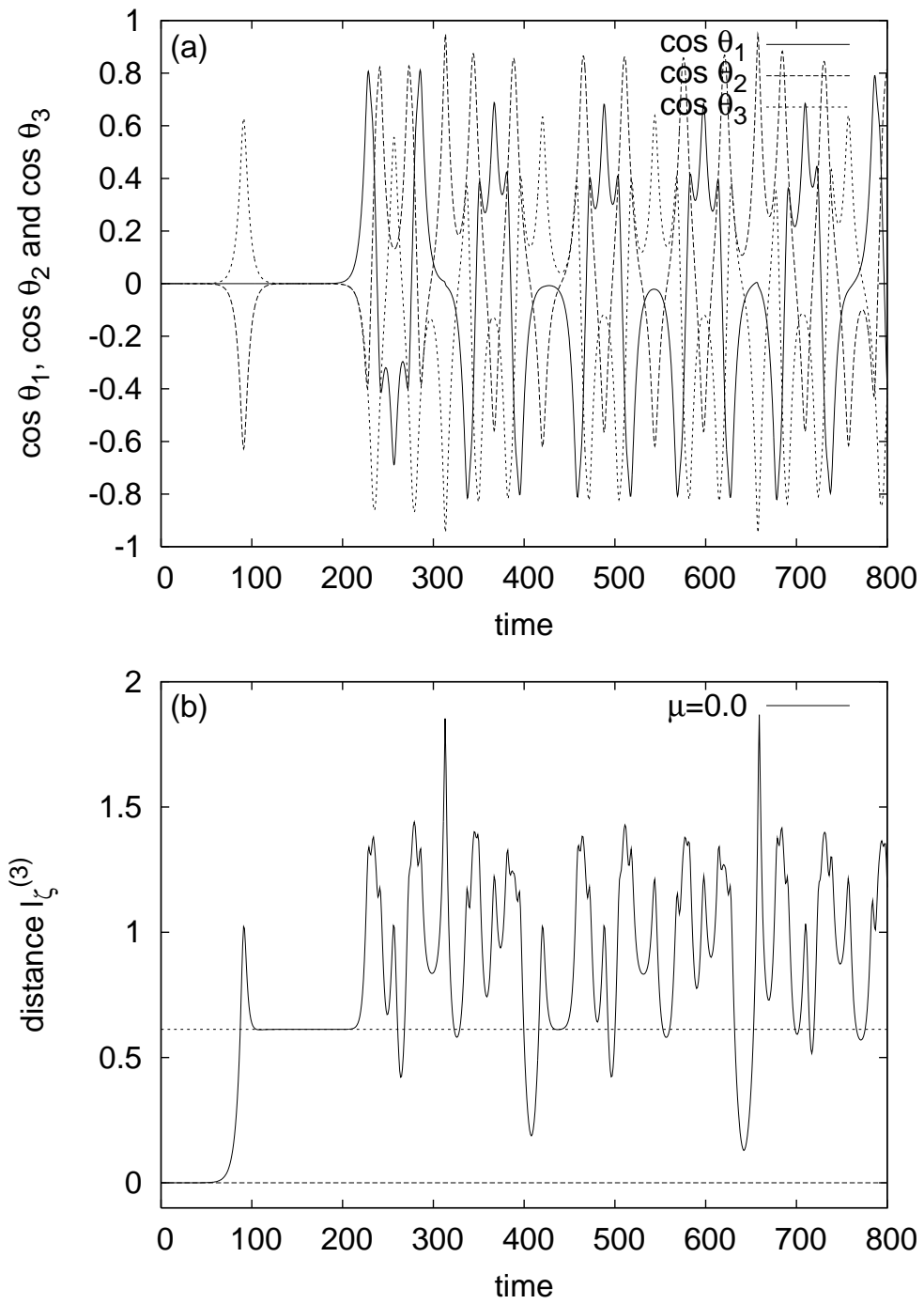

Figure 2: (a) Evolutions of $\cos \Theta_{1}, \cos \Theta_{2}$ and $\cos \Theta_{3}$, and (b) the distance $l_{\zeta}^{(3)}$ for the perturbed unstable 3 -ring at the equator when $\left(\Gamma_{1}, \Gamma_{2}\right)=(-0.2 \pi,-0.2 \pi)$. The dashed lines in (b) represent the value of 0 and $\frac{\sqrt{6}}{4}$ respectively. The initial unstable direction is $\mu=0.0$. 

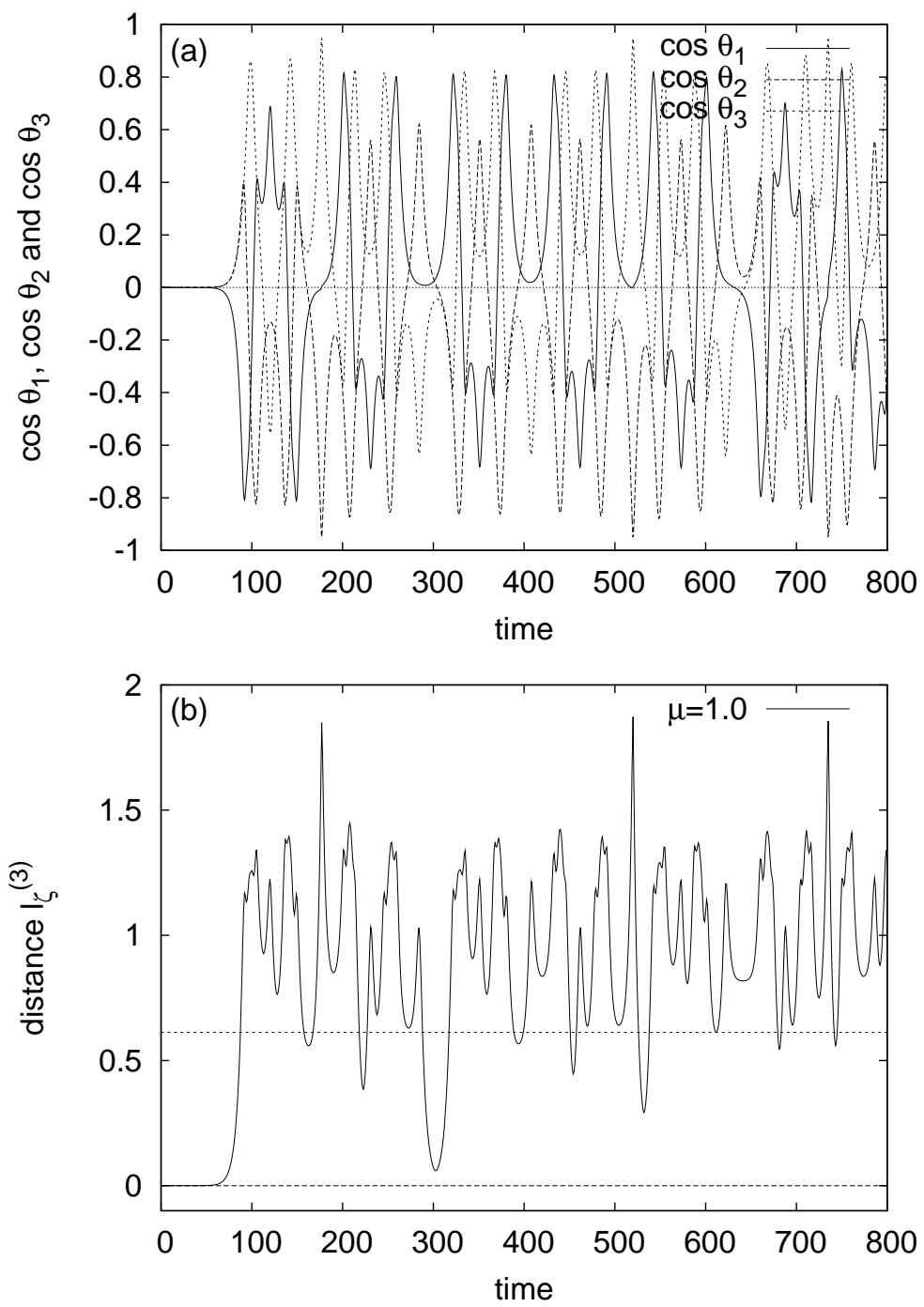

Figure 3: (a) Evolutions of $\cos \Theta_{1}, \cos \Theta_{2}$ and $\cos \Theta_{3}$, and (b) the distance $l_{\zeta}^{(3)}$ for the perturbed unstable 3 -ring at the equator when $\left(\Gamma_{1}, \Gamma_{2}\right)=(-0.2 \pi,-0.2 \pi)$. The dashed lines in (b) represent the value of 0 and $\frac{\sqrt{6}}{4}$ respectively. The initial unstable direction is $\mu=1.0$. The evolutions repeat the similar pattern and the distance $l_{\xi}^{(3)}$ passes in the neighborhood of 0 or $\frac{\sqrt{6}}{4}$ when both of the evolutions enter in the vicinity of the zero-line. 

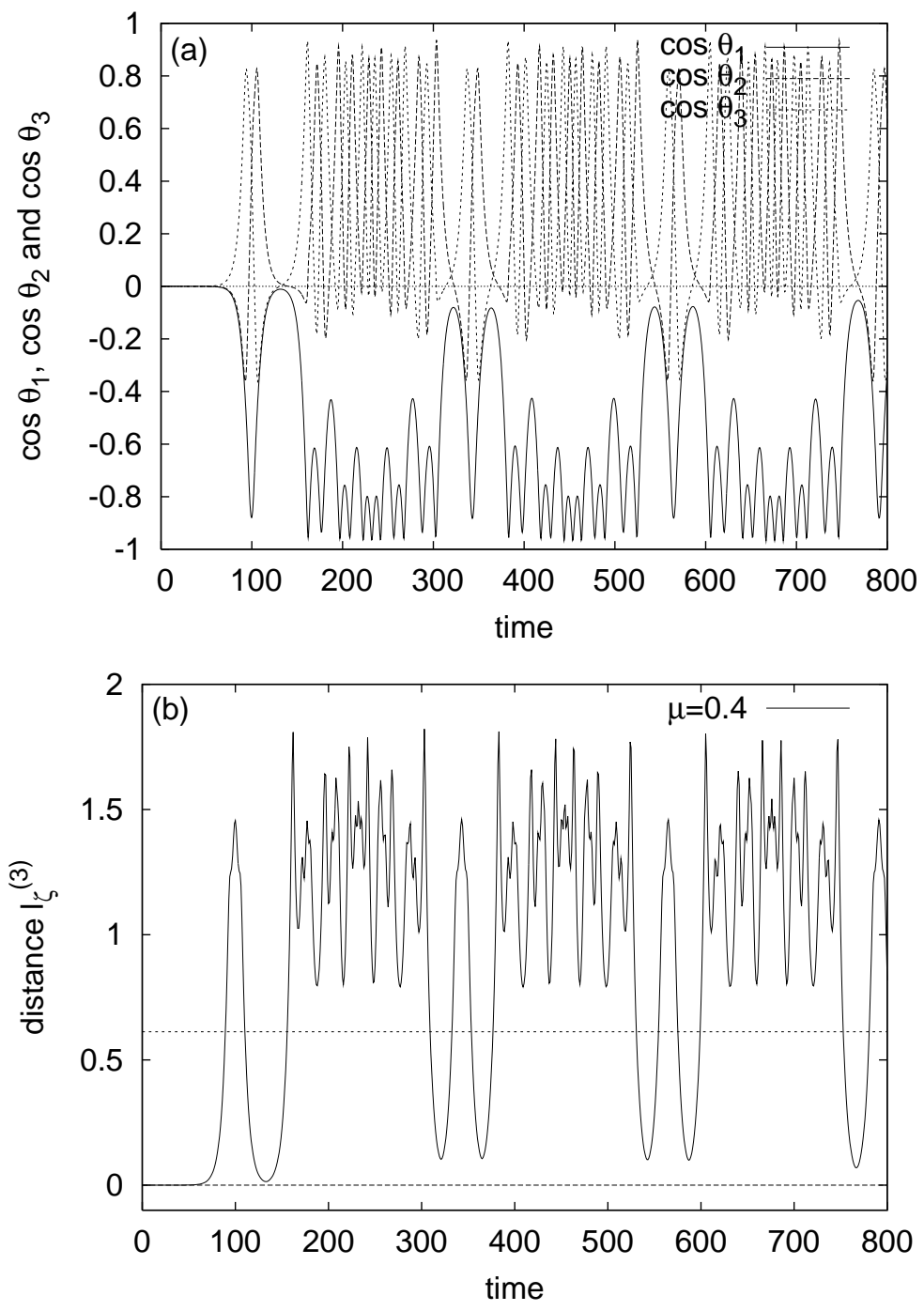

Figure 4: (a) Evolutions of $\cos \Theta_{1}, \cos \Theta_{2}$ and $\cos \Theta_{3}$, and (b) the distance $l_{\zeta}^{(3)}$ for the perturbed unstable 3 -ring at the equator when $\left(\Gamma_{1}, \Gamma_{2}\right)=(-0.2 \pi,-0.2 \pi)$. The dashed lines in (b) represent the value of 0 and $\frac{\sqrt{6}}{4}$ respectively. The initial unstable direction is $\mu=0.4$. The distance approaches zero periodically. 

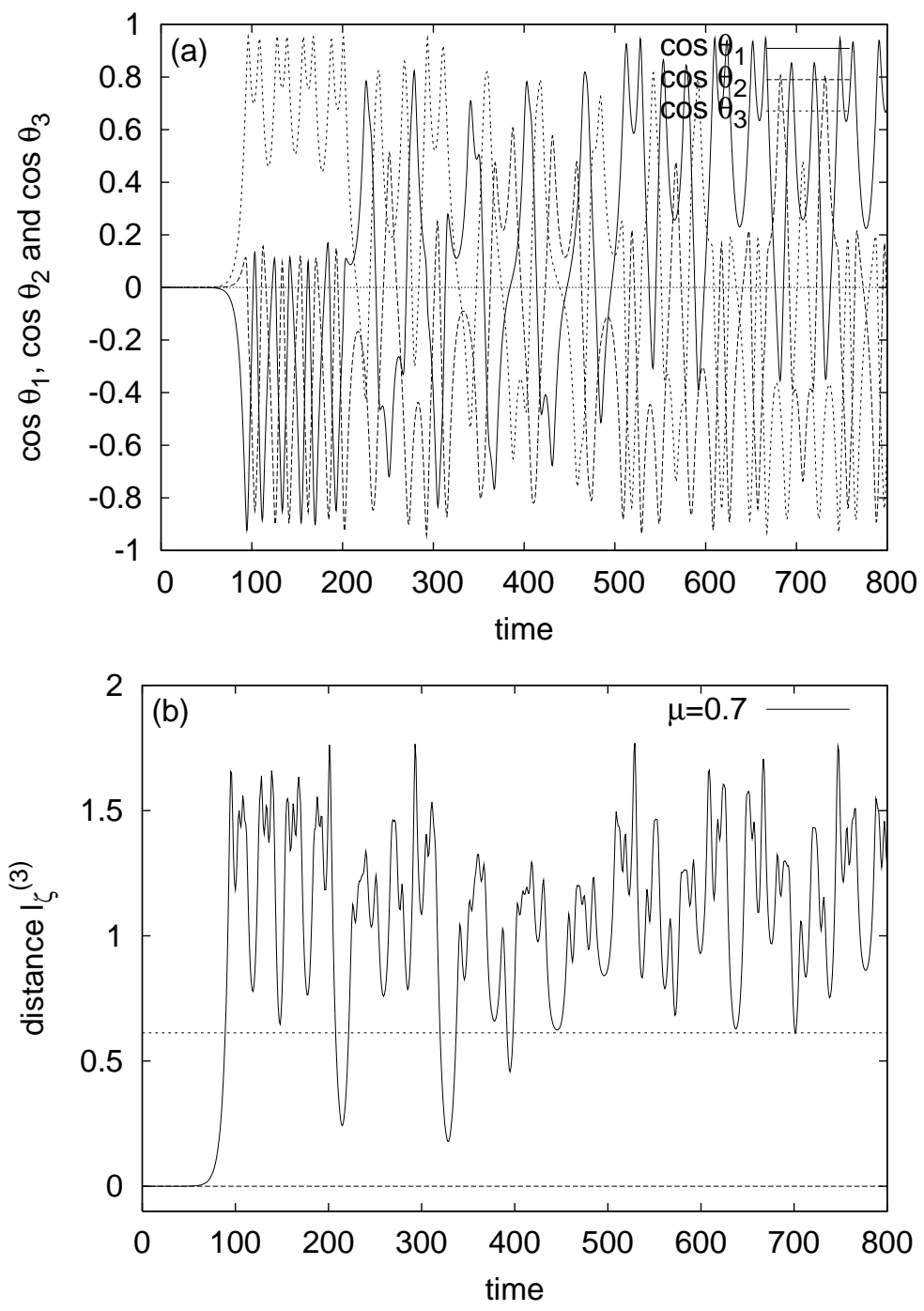

Figure 5: (a) Evolutions of $\cos \Theta_{1}, \cos \Theta_{2}$ and $\cos \Theta_{3}$, and (b) the distance $l_{\zeta}^{(3)}$ for the perturbed unstable 3 -ring at the equator when $\left(\Gamma_{1}, \Gamma_{2}\right)=(-0.2 \pi,-0.2 \pi)$. The dashed lines in (b) represent the value of 0 and $\frac{\sqrt{6}}{4}$ respectively. The initial unstable direction is $\mu=0.7$. The distance occasionally approaches in the neighborhood of 0 and $\frac{\sqrt{6}}{4}$, but the evolutions look very irregular. 
(a)
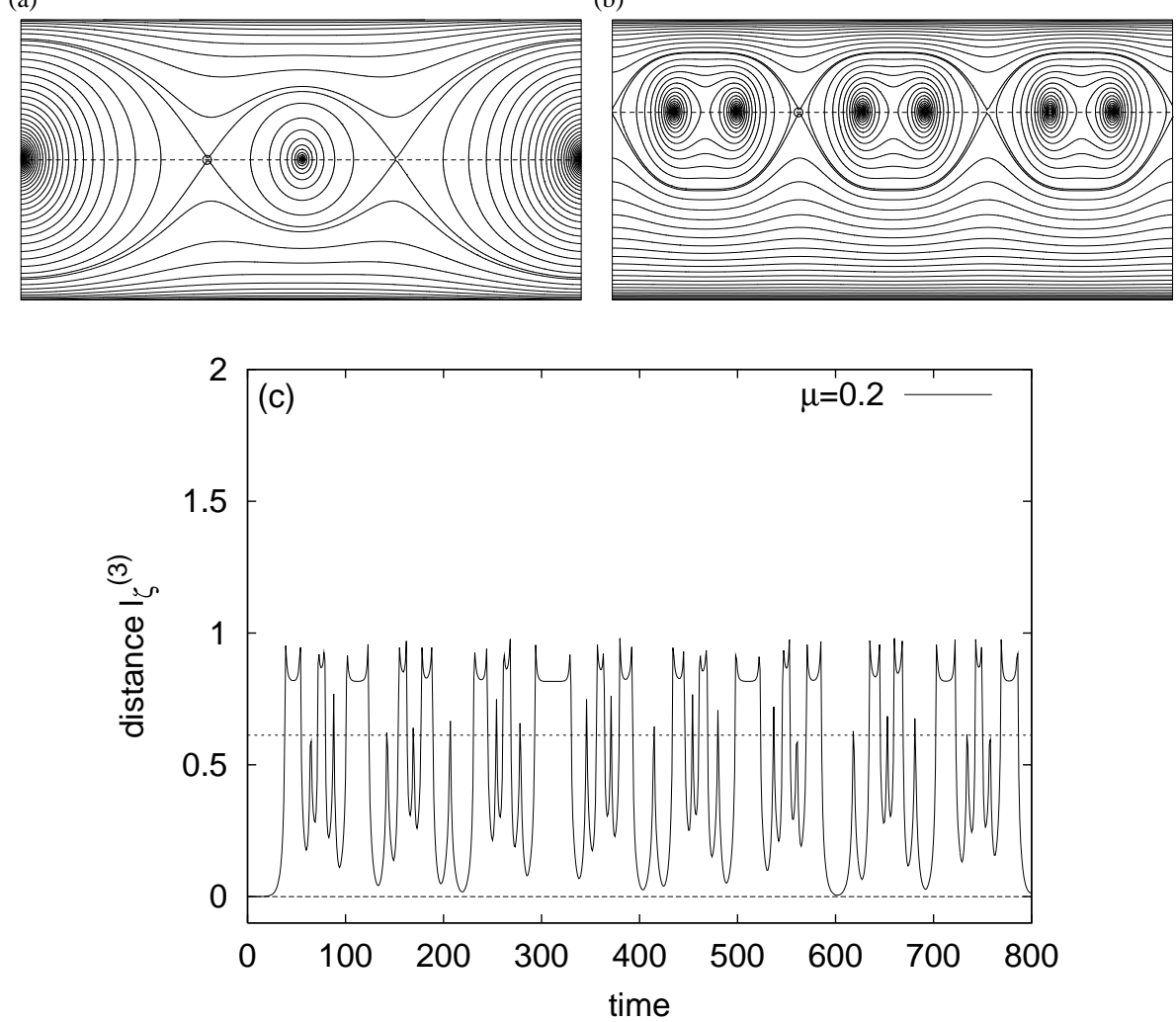

Figure 6: Contour plots of the projected Hamiltonians (a) $H_{\vec{\phi}_{1}^{ \pm}}$and (b) $H_{\vec{\psi}_{1}^{ \pm}}$in $\left(\Psi_{2}, \Theta_{2}\right)$-plane, when the unstable 3-ring is located at $\theta_{0}=\frac{\pi}{4}$ and $\left(\Gamma_{1}, \Gamma_{2}\right)=$ $(-0.3 \pi,-0.4 \pi)$. The circles in the figures denote the steady 3-ring. (c) Evolution of the distance $l_{\zeta}^{(3)}$ for $\mu=0.2$. The dashed lines represent the value of 0 and $\frac{\sqrt{6}}{4}$ respectively. 
(a)

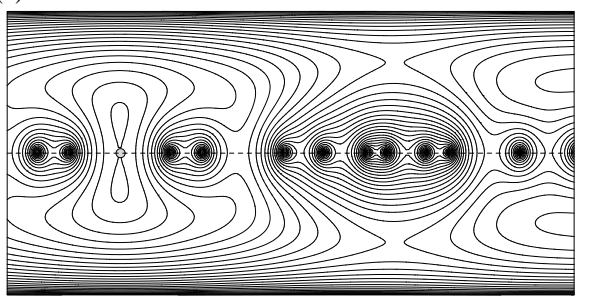

(b)

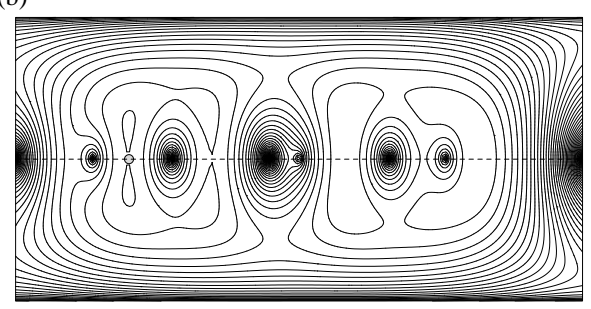

Figure 7: Contour plots of the projected Hamiltonians (a) $H_{\vec{\phi}_{2}^{ \pm}}$and (b) $H_{\vec{\psi}_{2}^{ \pm}}$in $\left(\Psi_{2}, \Theta_{2}\right)$-plane, when the 5-ring is located at the equator for $\left(\Gamma_{1}, \Gamma_{2}\right)=(0.2 \pi, 0.2 \pi)$. The circles in the figures denote the steady 5-ring.

restrict the range of $\Theta_{2}$ by using the constraint conditions, due to $0 \leq \Theta_{i} \leq \pi$ for $i=1, \cdots, 5$.

Figure 7 shows the contour plot of the projected Hamiltonians $H_{\vec{\phi}_{2}^{ \pm}}$and $H_{\vec{\psi}_{2}^{ \pm}}$ in $\left(\Psi_{2}, \Theta_{2}\right)$-plane for $\left(\Gamma_{1}, \Gamma_{2}\right)=(0.2 \pi, 0.2 \pi)$, in which the largest eigenvalue $\lambda_{2}^{+}$ becomes unstable and the second largest $\lambda_{1}^{ \pm}$are neutrally stable. The projected unstable and stable manifolds departing from the 5-ring return to the original position, which indicates that the iso-surface of the Hamiltonian has the homoclinic connection in $\mathbb{P}_{5}$. On the other hand, when both of the eigenvalues $\lambda_{1}^{+}$and $\lambda_{2}^{+}$ become unstable, we plot the iso-surface of the projected Hamiltonians (a) $H_{\vec{\phi}_{2}^{ \pm}}$, (b) $H_{\vec{\psi}_{2}^{ \pm}}$, (c) $H_{\vec{\phi}_{1}^{ \pm}}$and (d) $H_{\vec{\psi}_{1}^{ \pm}}$in Figure 8. In this case, the unstable and the stable manifolds no longer correspond to each other, nor do they connect to other 5-ring configurations.

We see the equilibrium-like points along the line $\Theta_{2}=\frac{\pi}{2}$ in the projected spaces, but in fact they are not real equilibria except the original 5-ring. However, since the homoclinic connection we observe in the former case is a local structure, we expect that the homoclinic structure of the iso-surface of the Hamiltonian in the neighborhood of the original 5-ring affect the evolution of the perturbed 5-ring, even if the projection method is just a restriction of the system based on the local expansion. On the contrary, as for the latter case, since we can hardly see any local structure, the projection method fails to give any useful information of the structure of the Hamiltonian.

\section{$5 \quad$ Summary and remark}

We have investigated the motion of the $N$-ring on the sphere when the effect of the rotation is approximated locally by the fixed pole vortices. Since the linear stability analysis o the $N$-ring gives the explicit representation of the eigenvectors $\lambda_{p}^{ \pm}$and their linearly independent eigenvectors $\vec{\psi}_{p}^{ \pm}$and $\vec{\phi}_{p}^{ \pm}$for $p=1, \cdots, M$, it is possible to express the orbit of the vortex points in the phase space $\mathbb{P}_{N}$ as the linear combination of the eigenvectors. The linear combination provides us with the projection conditions, with which we can restrict the $2 N$-dimensional dynamical system to the collection of the two-dimensional systems spanned by the pair of the unstable and stable eigenvectors. Thus projecting the iso-surface of the Hamiltonian to the restricted phase spaces, we observe the global structure of the iso-surface, in which the orbit of the vortex points exists.

Since the projection method just restrict the high-dimensional dynamical system to the two-dimensional eigenspaces, it is generally difficult to extract some infor- 
(a)

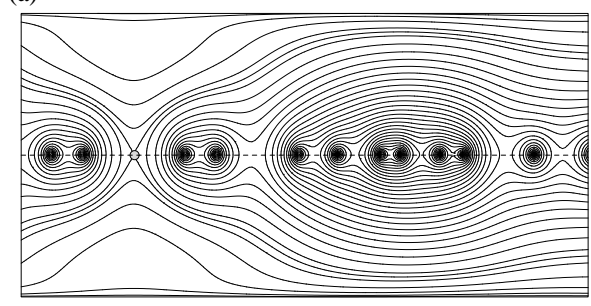

(c)

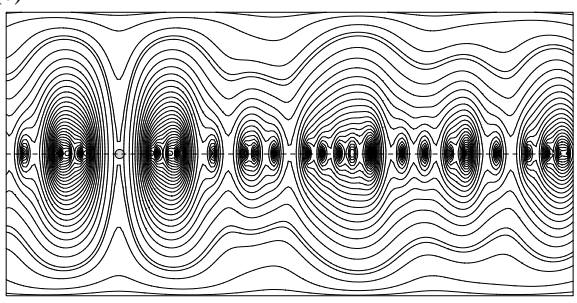

(b)

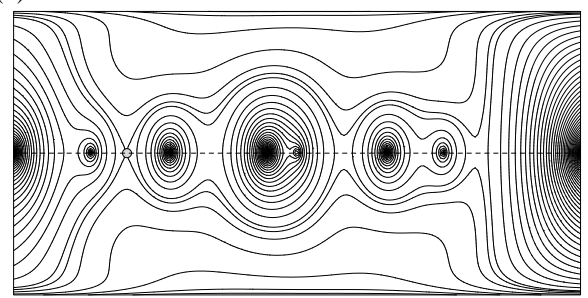

(d)

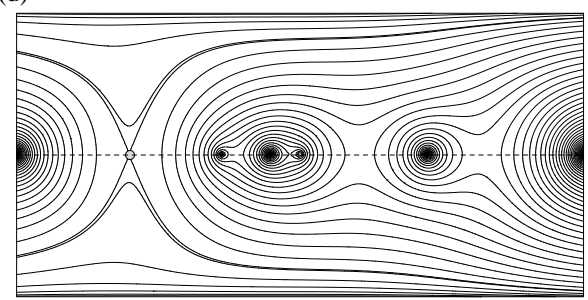

Figure 8: Contour plots of the projected Hamiltonians (a) $H_{\vec{\phi}_{2}^{ \pm}}$, (b) $H_{\vec{\psi}_{2}^{ \pm}}, H_{\vec{\phi}_{1}^{ \pm}}$and $H_{\vec{\psi}_{1}^{ \pm}}$in $\left(\Psi_{2}, \Theta_{2}\right)$-plane, when the 5 -ring is located at the equator for $\left(\Gamma_{1}, \Gamma_{2}\right)=$ $(-0.1 \pi,-0.1 \pi)$. The circles in the figures denote the steady 5 -ring.

mation of the original system by the restriction. However, in some specific cases of the 3-ring and the 5-ring, the restriction indicates the local and global structures of the iso-surface of the Hamiltonian help us characterize the unstable evolution; Applying the projection method to the 3-ring at the equator with the same pole vortices, we show the existence of the invariant two-dimensional dynamical system, in which there exists the heteroclinic orbit. In addition, since the iso-surface has the heteroclinic connection, when the unstable 3-ring is perturbed slightly, the periodic and the non-trivial recurrent evolutions are observed. However, since the co-dimension of the orbit in the high dimensional iso-surface of the Hamiltonian is high, the unstable 3-ring sometimes shows the irregular behavior depending on the initial direction of the perturbation. In the similar manner, we apply the projection method to the 5-ring case and observe the homoclinic connection of the iso-surface of the Hamiltonian.

Finally, we remark the application of the projection method to the case of even vortex points which has already been studied in [19]. As we stated in the introduction, the multiplicity of the largest eigenvalues $\lambda_{M}^{ \pm}$is different from the odd case. That is to say, the largest eigenvalues $\lambda_{M}^{ \pm}$have just one eigenvectors $\vec{\psi}_{M}^{ \pm}\left(=\vec{\phi}_{M}^{ \pm}\right)$. In the paper [19], we successfully reduced the $2 N$-dimensional dynamical system to the planar system with the alternately pairing condition introduced heuristically from the symmetry of the eigenvectors $\vec{\psi}_{M}^{ \pm}$. As a matter of fact, the alternately pairing symmetry is justified by the projection method; When we apply the projection to the even case, the orbit of the vortex points $\vec{x}$ can be similarly expressed by the linear combination of the eigenvectors $\vec{\phi}_{p}^{ \pm}, \vec{\psi}_{p}^{ \pm}$for $p=1, \cdots, M-1, \vec{\psi}_{M}^{ \pm}$and the complementary vectors $\vec{\zeta}^{ \pm}$as follows:

$$
\vec{x}=\vec{x}_{0}+a_{M} \vec{\psi}_{M}^{+}+b_{M} \vec{\psi}_{M}^{-}+\sum_{p=1}^{M-1}\left(a_{p} \vec{\psi}_{p}^{+}+b_{p} \vec{\psi}_{p}^{-}+c_{p} \vec{\phi}_{p}^{+}+d_{p} \vec{\phi}_{p}^{-}\right)+e \vec{\zeta}^{+}+f \vec{\zeta}^{-} .
$$

The constraint condition to project the orbit on the two-dimensional eigenspace spanned by $\vec{\psi}_{M}^{ \pm}$are provided by solving $a_{p}=b_{p}=c_{p}=d_{p}=0$ for $p=1, \cdots, M-1$, 
and $e=f=0$. Solving these equations, we obtain the alternately pairing condition. Thus, unlike the odd case, the projection method always reduces the even $N$-ring system to the two-dimensional invariant dynamical system regardless of the position of the ring and the strengths of the pole vortices.

\section{References}

[1] G.K. Batchelor, An introduction to fluid dynamics, Cambridge Univ. Press, Cambridge, 1967.

[2] S. Boatto and H.E. Cabral, Nonlinear stability of a latitudinal ring of pointvortices on a nonrotating sphere, SIAM J. Appl. Math., 64(2003), pp. 216-230.

[3] D.G. Dritschel, The stability and energetics of co-rotating uniform vortices, J. Fluid Mech., 358 (1985), pp.95-134.

[4] R. Kidambi and P.K. Newton, Motion of three point vortices on a sphere, Physica D, 116 (1998), pp.95-134.

[5] R. Kidambi and P.K. Newton, Collapse of three vortices on a sphere, Nouvo Cimento, 22C (1999), pp. 779-791.

[6] R. Kidambi and P.K. Newton, Streamline topologies for integrable vortex motion on a sphere, Physica D, 140 (2000), pp. 95-125.

[7] F. Kirwan, The topology of reduced phase space of the motion of vortices on a sphere, Physica D, 30 (1998), pp. 99-123.

[8] Y. Kimura and H. Okamoto, Vortex motion on a sphere, J. Phys. Soc. Japan, 56 (1987), pp. 4203-4206.

[9] L.G. Kurakin, On nonlinear stability of the regular vortex systems on a sphere, Chaos, 14 (2004), pp.592-602.

[10] F. Laurent-Polz, Point vortices on the sphere: A case with opposite vortices, Nonlinearity, 15 (2003), pp. 143-171.

[11] F. Laurent-Polz, Relative periodic orbits in point vortex systems, Nonlinearity, 17 (2004) pp.1989-2013.

[12] C. Lim, Relative equilibria of symmetric n-body problems on a sphere: inverse and direct results, Comm. Pure. Appl. Math., 51 (1998), pp. 341-371.

[13] C. Lim, J. Montaldi, and M. Roberts, Relative equilibria of point vortices on the sphere, Phys. D, 148 (2001), pp.97-135.

[14] P.K. Newton, The N-vortex problem, Analytical techniques, Springer-Verlag, New York, 2001.

[15] S. Pekarsky and J.E. Marsden, Point vortices on a sphere: Stability of relative equilibria, J. Math. Phys., 39 (1998), pp. 5894-5907.

[16] L.M. Polvani and D.G. Dritschel, Wave and vortex dynamics on the surface of a sphere, J. Fluid Mech., 255 (1993), pp.35-64.

[17] T. Sakajo, The motion of three point vortices on a sphere, Japan J. Indust. Appl. Math., 16 (1999), pp. 321-347.

[18] T. Sakajo, Motion of a vortex sheet on a sphere with pole vortices, Phys. Fluids, 16 (2004), pp. 717-727. 
[19] T. Sakajo, Transition of global dynamics of a polygonal vortex ring on a sphere with pole vortices, Physica D, 196 (2004), pp. 243-264.

[20] T. Sakajo, Motion of unstable polygonal ring of vortex points on sphere with pole vortices, submitted to Proc. IUTAM (2004).

[21] A. Souliére and T. Tokieda, periodic motions of vortices on surfaces with symmetry, J. Fluid Mech., 460 (2002), pp.83-92. 